\title{
Effect of oil replacement in mayonnaise by chia (Salvia hispanica L) mucilage
}

\author{
Sibele Santos Fernandesa* and Myriam de las Mercedes Salas-Mellado \\ Laboratory of Food Technology, School of Chemistry and Food, Federal University of Rio Grande, Av Italy km 8, Carreiros 96203-900, Brazil
}

\begin{abstract}
Chia mucilage, composed mainly of dietary fibers, is obtained by immersing chia seeds in water, where the formation of a mucilaginous transparent gel occurs due to the presence of hydrophilic molecules that combine with water. This gel has many properties, among them the emulsifier. Mayonnaise is one of the most consumed condiments, however it contains 70 to $80 \%$ of oil. In addition, currently, it exists a growing demand for healthier foods with reduced fat. The objective of this work was to verify the effect of adding the chia mucilage in the substitution of part of the oil in mayonnaise. The chia mucilage was extracted after 2 hours of hydration of the chia seeds through vacuum pump and single sieve, and finally lyophilized. The mayonnaise was prepared with soybean oil, dehydrated egg yolk, vinegar, salt, sugar and chia mucilage. The effect was tested on a mayonnaise with the $15 \%$ of the oil substituted by the addition of $0.5 \%, 1 \%$ and $1.5 \%$ of chia mucilage in the formulation, through the properties of water activity, $\mathrm{pH}$, color, stability and texture parameters. The water activity increased, as occurred with the addition of chia mucilage, varying from 0.85 to 0.90 . The mayonnaise containing $1 \%$ of mucilage showed $\mathrm{pH}(3.44)$ statistically equal to that of standard mayonnaise (3.48) and lower than the other formulations. As for stability, only mayonnaise added with $0.5 \%$ chia mucilage was statistically different from the others, presenting lower stability ( $72.85 \%)$. In relation to texture, higher values of firmness and consistency and lower values of cohesiveness and viscosity index were obtained for the mayonnaise containing $1 \%$ mucilage. With this study, it is verified that the substitution of $15 \%$ of the oil in mayonnaises can be compensated by the addition of $1 \%$ of lyophilized chia mucilage, due to its excellent emulsifying capacity.
\end{abstract}

\section{Introduction}

Fat substitutes are substances that can be used in different foods to mimic the textured and sensory attributes provided by fat, but provide a considerably smaller amount of calories, and can be based on proteins, carbohydrates or fats [1]. However, it is often difficult to preserve the desirable sensory properties of the food product by reducing or replacing the fat with respect to the breaking strength, texture, moisture content and mouth feel [2].

Currently, there are several new fat replacements to meet the demands associated with the current trend of low-fat foods [3]. In this context, a fat substitute that presents great potential and already is being applied in the fat substitution in bakery products, such as bread and cakes $[4,5]$, is the chia mucilage, because its functional characteristics and low fat content.

Chia seed (Salvia hispânica L.), native to southern Mexico and northern Guatemala, was traditionally one of the basic elements in the diet of Central American civilizations in the pre-Columbian epoch and was used as an offering to the god. Today chia is being re-introduced into diets because be rich source of essential fatty acids, dietary fiber and proteins [6].

In the presence of water, the chia seed exudes a transparent mucilaginous gel, composed essentially of soluble fibers. Chia mucilage is formed by the addition of water to chia seeds, being formed mainly by soluble fibers [4] and features characteristics, such as thickeners, stabilizers and film/coating agents, that allows its incorporation in foods $[6,7]$. The chia mucilage is a highly branched tetrasaccharide, being formed by a main chain constituted by units of $(1 \rightarrow 4)-\beta$-Dxylopyranosyl-( $1 \rightarrow 4)$ - $\alpha$-D-glucopyranosyl- $(1 \rightarrow 4)-\beta$-D-xylopyranosyl with branches of 4-O-methyl- $\alpha$-D-glucuronic acid at the O-2 position of $\beta$-D-xylopyranosyl in the main chain [8].
There are still few applications of chia mucilage. Spada et al. [9] produced soy-based desserts using chia mucilage in the formulation instead of the thickening agent. Campos et al. [10] evaluated the potential of chia mucilage in the total substitution of emulsifiers and stabilizers in ice cream. Recently, Fernandes and Salas-Mellado [4] andSalgado-Cruz et al. [11] used chia mucilage in wheat bread formulation. In addition, also in the area of baking, chia mucilage was used as a substitute for fat in cakes by Borneo et al. [12], Felisberto et al. [5] and Fernandes and Salas-Mellado [4]. Menga et al. [13] added chia mucilage together with rice flour in order to develop gluten free pasta.

Increasing consumer interest in low-fat food products is the basis for new product development studies by incorporating or replacing certain ingredients, assessing the influence on the quality and taste of food emulsions. The use of different types of emulsifiers, thickeners and fat substitutes has been studied by some researchers [14]. As previously mentioned, there are still no applications of chia mucilage chia in emulsified products. Among these, mayonnaise is one of the most consumed condiments in the world, since it is a food emulsion used both as an individual consumption as well as an ingredient in sauces. Mayonnaise, traditionally, is an emulsion prepared by the careful mixing of egg yolk, vinegar, oil and spices, especially mustard [15]. The mayonnaise produced in this way, typically, contains high fat (70-80\%) and cholesterol content [16].

${ }^{\star}$ Correspondence to: Sibele Santos Fernandesa, Laboratory of Food Technology, School of Chemistry and Food, Federal University of Rio Grande, Av Italy km 8 Carreiros 96203-900, Brazil, Tel: 0555332336974; E-mail: sibelecti@hotmail.com

Key words: chia seed, emulsion, replacement, texture, stability

Received: April 9, 2018; Accepted: May 11, 2018; Published: May 16, 2018 
The reduction of fat in mayonnaise can have an impact on its sensorial, physical and technological characteristics, being, mainly, the rheological and textural behavior are the most influenced and focus on storage stability. In addition, the taste perception of reduced fat can also be significantly altered. Because of this, the food industries face challenges as no single ingredient can mimic the characteristics [14]. The objective of this work was to study the effect of the different substitution of oil in mayonnaise by addition of lyophilized mucilage chia in different concentrations and to evaluate the physical and technological characteristics, in order to verify the amount of substitution that can be made without affecting the properties of mayonnaise.

\section{Material and methods}

\section{Material}

Chia seeds ( $35.7 \mathrm{~g} / 100 \mathrm{~g}$ lipids, $4.9 \mathrm{~g} / 100 \mathrm{~g}$ ash, $19.6 \mathrm{~g} / 100 \mathrm{~g}$ protein, $17.2 \mathrm{~g} / 100 \mathrm{~g}$ crude fiber, $22.6 \mathrm{~g} / 100 \mathrm{~g}$ carbohydrates) were kindly provided by Dubai - Industry and Commerce of Food Products Ltda, located in Ijuí/RS, and were packed in hermetic plastic vessels and stored at $5^{\circ} \mathrm{C}$ until use. The dehydrated egg yolk was kindly donated by NaturOvos, located in Salvador do Sul/RS. The other ingredients, such as soybean oil, vinegar, salt and sugar were purchased in local shops. All reagents used in the chemical determinations were from analytical grade.

\section{Methods}

\section{Extraction of mucilage}

Water was add at seed chia in the ratio of 1:40 (seed:distilled water) shaking at $150 \mathrm{rpm}$ in a shaker (Cientec, model CT-712RNT, Brazil) for $2 \mathrm{~h}$ at room temperature. Thereafter, the mucilage formed was separated from the chia seed by 5 filtration using a vacuum pump and sieve (18mesh). The filtrate was centrifuged (Hanil, model Supra $22 \mathrm{~K}$, Korea) at $11600 \mathrm{xg}$ for $20 \mathrm{~min}$. The supernatant (mucilage) was frozen in an ultrafreezer at $-80^{\circ} \mathrm{C}$ and later freeze-dried (Liobrás, model L108, Brazil).

The lyophilized chia mucilage presented an extraction yield of $5.81 \%$, and a composition in dry basis of $10.63 \mathrm{~g} / 100 \mathrm{~g}$ of protein, 8.79 $\mathrm{g} / 100 \mathrm{~g}$ of lipids, $2.05 \mathrm{~g} / 100 \mathrm{~g}$ of ash and $78.53 \mathrm{~g} / 100 \mathrm{~g}$ of carbohydrates [4]. And, it was wrapped in plastic containers and maintained at $-18{ }^{\circ} \mathrm{C}$ for later use.

\section{Preparation of mayonnaise}

The mayonnaise formulation showed in Table 1 was elaborated according to Kishk and Elsheshetawy [17], with modifications. Soybean oil was reduced of $15 \%$ in relation to the control formulation. In order to verify the amount of chia mucilage that must be added to supply the reduced $15 \%$ of oil, different concentrations of lyophilized chia mucilage at different levels was added: $0.5 \%$ (addition of $0.105 \mathrm{~g}$ chia mucilage in substitution to $21 \mathrm{~g}$ of the oil, $1.0 \%$ (addition of $0.210 \mathrm{~g}$ chia mucilage in to $21 \mathrm{~g}$ of the oil and $1.5 \%$ (addition of $0.315 \mathrm{~g}$ chia mucilage in substitution to $21 \mathrm{~g}$ of the oil.

First, part of the water was used to hydrate the lyophilized chia mucilage. The dehydrated egg yolk, rest of the water and the vinegar were mixed. Subsequently, salt and sugar were added. In a planetary mixer (KitchenAid), soybean oil was slowly added through a burette. The hydrated mucilage was added after all the oil had been added. The mayonnaise samples were stored in polyethylene pots with a screw cap, identified, dated and stored under refrigeration $\left(\right.$ at $\left.4^{\circ} \mathrm{C}\right)$ until the analyses were carried out.
Table 1. Formulation (g) of control mayonnaise and mayonnaises with replacement of oil by lyophilized chia mucilage

\begin{tabular}{|l|c|c|c|c|}
\hline Ingredients (g) & Control & MOCM-A & MOCM-B & MOCM-C \\
\hline Soybean oil & 140.0 & 119.0 & 119.0 & 119.0 \\
\hline Dehydrated egg yolk & 16.0 & 16.0 & 16.0 & 16.0 \\
\hline Vinegar (Acetic acid 4.5\%) & 9.0 & 9.0 & 9.0 & 9.0 \\
\hline Sugar & 4.0 & 4.0 & 4.0 & 4.0 \\
\hline Salt & 3.0 & 3.0 & 3.0 & 3.0 \\
\hline Water & 18.000 & 38.895 & 38.790 & 38.685 \\
\hline Lyophilized chia mucilage & - & 0.105 & 0.210 & 0.315 \\
\hline Total & 190.0 & 190.0 & 190.0 & 190.0 \\
\hline
\end{tabular}

MOCM-A, MOCM-B and MOCM-C: formulations with $0.5,1.0$ and 1,5\% addition of lyophilized mucilage in relation the $15 \%$ reduced of oil, respectively

\section{Evaluation of the mayonnaises}

Determination of water activity: Samples of mayonnaise with different concentrations of chia mucilage were evaluated for water activity (Aw) through the LabTouch Novasina (Novasina, Model LabTouch, Switzerland) at room temperature.

Stability of the emulsion: The stability of the mayonnaises was determined using the methodology of Mun et al. [18]. The mass of the precipitated fraction was measured and stability was determined by Equation 1, where F0 represents the sample mass and F1 represents the precipitated mass.

\section{Stability $(\%)=$ F1/F0 $\times 100(1)$}

Determination of $\mathbf{p H}$ : The $\mathrm{pH}$ values of mayonnaise samples were measured at a room temperature through a potentiometer (model Q400AS, Quimis, Brazil).

Texture: The parameters evaluated were: firmness, consistency, cohesiveness and viscosity index. It was used a TA-XT2 texturometer (Stable Micro Systems, UK), provided with back extrusion software. The samples were carefully poured into $150 \mathrm{~mL}$ cylindrical containers (60 $\mathrm{mm}$ internal diameter and $80 \mathrm{~mm}$ height), up to the $125 \mathrm{~mL}$ mark. Compression was done with a disc of $35 \mathrm{~mm}$ in diameter and the applied cycle consisted of constant speed of $1 \mathrm{~mm} / \mathrm{s}$, until a depth of 40 $\mathrm{mm}$ in the sample. From the resulting force-time curve, the values of firmness, consistency, cohesiveness and viscosity index were obtained.

Color: The color analysis were determined in mayonnaises samples using a Colorimeter (Minolta, model CR400, Japan), through the color parameters $L^{\star}, a^{\star}$ and $b^{\star}$. The instrument was calibrated before each analysis with white and black standard tiles. The analysis was based on the CIE $\mathrm{L}^{*} \mathrm{a}^{*} \mathrm{~b}^{*}$ system that were defined by the CIE (International Commission on Illumination) in 1976 for determining the $L^{*}$ (lightness), $\mathrm{a}^{\star}$ and $\mathrm{b}^{\star}$ (chromaticity coordinates) values. The hue angle or $\mathrm{h}$ was also calculated, according to Equation 2.

$\mathrm{h}=\tan -1 \mathrm{~b}^{\star} / \mathrm{a}^{*}(2)$

\section{Statistical analysis}

All determinations were done at least in triplicate. The data were compared using analysis of variance (ANOVA) and the average values obtained were compared using Tukey's and t-Student test through the Statistica 5.0 software (Statsoft, USA), with statistical significance $(a)$ set at $\mathrm{p}<0.05$.

\section{Results and discussion}

\section{Water activity}

Table 2 shows the water activity, $\mathrm{pH}$, stability and texture parameters of the control mayonnaise and the mayonnaise with replacement of oil by addition lyophilized chia mucilage. 
Table 2. Water activity, $\mathrm{pH}$, stability and texture parameters for control mayonnaise and added chia mucilage

\begin{tabular}{|c|c|c|c|c|c|c|c|}
\hline \multirow[b]{2}{*}{ Formulation } & \multirow[b]{2}{*}{ Aw } & \multirow[b]{2}{*}{ pH } & \multirow[b]{2}{*}{ Stability (\%) } & \multicolumn{4}{|c|}{ Texture } \\
\hline & & & & Firmness (g) & Consistency (g.seg) & Cohesiveness (g) & $\begin{array}{l}\text { Viscosity index } \\
\text { (g.seg) }\end{array}$ \\
\hline Control & $0.851 \pm 0.003^{\mathrm{d}}$ & $3.48 \pm 0.02^{\mathrm{c}}$ & $98.95 \pm 1.13^{\mathrm{a}}$ & $141.91 \pm 1.50^{\mathrm{b}}$ & $4949.52 \pm 145.87^{\mathrm{b}}$ & $-150.75 \pm 6.92^{b}$ & $-458.97 \pm 9.06^{b}$ \\
\hline MOCM-A & $0.873 \pm 0.003^{\mathrm{c}}$ & $3.63 \pm 0.02^{\mathrm{a}}$ & $72.86 \pm 0.86^{\mathrm{b}}$ & $118.16 \pm 3.42^{\mathrm{c}}$ & $4198.07 \pm 147.16^{\mathrm{c}}$ & $-123.10 \pm 5.48^{\mathrm{a}}$ & $-397.65 \pm 7.32^{\mathrm{a}}$ \\
\hline MOCM-B & $0.886 \pm 0.007^{\mathrm{b}}$ & $3.44 \pm 0.02^{\mathrm{c}}$ & $98.86 \pm 0.35^{\mathrm{a}}$ & $211.75 \pm 11.22^{\mathrm{a}}$ & $7331.42 \pm 32.54^{\mathrm{a}}$ & $-226.41 \pm 10.55^{c}$ & $-675.65 \pm 7.22^{c}$ \\
\hline МOCM-C & $0.900 \pm 0.001^{\mathrm{a}}$ & $3.58 \pm 0.01^{\mathrm{b}}$ & $97.05 \pm 0.81^{\mathrm{a}}$ & $127.53 \pm 7.40^{\mathrm{b}, \mathrm{c}}$ & $4538.57 \pm 214.82^{\mathrm{c}}$ & $-131.56 \pm 7.64^{a, b}$ & $-391.86 \pm 1.60^{\mathrm{a}}$ \\
\hline
\end{tabular}

MOCM-A, MOCM-B and MOCM-C: formulations with $0.5,1.0$ and 1,5\% addition of lyophilized mucilage in relation the $15 \%$ reduced of oil, respectively. Aw: activity water. Average of three values with standard deviation, same letter in the column indicates that there is no significant difference between the means by Tukey test $(\mathrm{p}<0.05)$

Water activity is important because it shows the water that is not attached to the substrate and therefore available for the proliferation of microorganisms [4]. According to Table 2, the water activity increased as the amount of mucilage increased in the mayonnaise and all samples showed values of this parameter higher than the control. Chia mucilage because it is formed by soluble fibers, presents high capacity of water retention, and in this work it was verified that the amount of free water increased with the addition of mucilage. However, the values found were still lower than in other studies, such as in mayonnaises developed with low-fat containing xanthan gum, guar gum and a combination of xanthan and guar gums [19].

\section{pH}

In mayonnaise, the growth of microorganisms is avoided by the high fat content and low water content, and at the same time, due to the presence of acetic acid, resulting in a low $\mathrm{pH}$ value [20]. The $\mathrm{pH}$ of mayonnaise can have a dramatic effect on the structure of the emulsion. At the average isoelectric $\mathrm{pH}$ of egg yolk proteins, the viscoelasticity and stability of mayonnaise are at their highest value. If the proteins on the surface of the droplets are highly loaded, it causes the inability to absorb any other protein and the droplets will repel one another, which would prevent flocculation. The viscoelasticity of mayonnaise is said to be highest at $\mathrm{pH} 3.9$ [15]. The critical $\mathrm{pH}$ that affects the useful life of mayonnaise is 4.1 , which means that when the $\mathrm{pH}$ is higher than 4.1 , the growth rate of the microorganisms in the system increases significantly [20].

The added mayonnaise of chia mucilage had higher $\mathrm{pH}$ than the control mayonnaise, with the exception of the MOCM-B formulation that did not differ significantly from the control sample. According to Gaonkar et al. [21] the normal pH of the mayonnaise is around 3.70, the MOCM-A formulation being the closest to this value.

\section{Stability}

The coalescence of the oil droplets, flocculation and formation of the cream are parameters that are associated to the stability of the emulsion. Many factors, such as frozen or dried egg yolk, added amount of salt and sucrose, oil concentration and purity, emulsion $\mathrm{pH}$ and addition of spices may contribute to emulsion instability. Low fat products, cream formation is avoided by addition of a thickening agent such as gums and starches [18].

From Table 2 it is possible to verify that the lowest amount of chia mucilage $(0.105 \mathrm{~g})$ was not able to maintain the stability of mayonnaise in relation to the control, promoting the coalescence of the oil droplets. Capitani et al. [22] obtained similar behavior in emulsions with low content chia mucilage. The authors justified this fact because of the low concentrations of emulsion polysaccharides, which favor faster rates of flocculation, coalescence and creams.

Thus, additions above $0.21 \mathrm{~g}$ of chia mucilage have the ability to simulate the stability of the $15 \%$ reduction of oil, being that the presence of gums, such as chia mucilage, might create a gel like structure that trap oil droplets, slow down their movements and raising the viscosity.

\section{Texture}

Through texture analysis, it is possible to imitate the action performed by the mouth. These tests are valuable, because they can show various characteristics, including the creaminess of the mayonnaise. The texture is also perceived outside the mouth (extra orally), before the beginning of its life, visual cues related to the items appearance of the item the information about its texture, while also can be used to capture the food, e.g., by stirring, spooning and cutting [23].

The parameters that involve texture are firmness, consistency, cohesiveness and viscosity index. Firmness is an indicator of resistance to penetration by a probe and will be greater as the force required for penetration increases. In addition, it reveals the solid properties of mayonnaise, such as cohesiveness and consistency, viscosity properties [20]. Consistency is obtained by the integral of the area of the graph formed by force versus distance until the maximum force observed and is associated with insertion of a spoon into a pot of mayonnaise.

The large amount of oil added to the mayonnaise formulation is what guarantees its high viscosity, and the larger the amount of oil added, the greater the amount of droplets formed, which will occupy more space in the aqueous phase, obstructing and hampering the flow. Firmness values were lower than those reported by Olosson et al. [23], whereas Raikos et al. [16] obtained lower values in the texture parameters. The MOCM-B formulation presented the highest texture parameters in comparison to the other formulations, although the formulations with oil reduction presented the same amount of oil, evidencing that the amount of mucilage influences the texture. This result is probably caused by increasing the viscosity of the emulsion due to the addition of chia mucilage to the formulation.

While the MOCM-A and MOCM-C formulations presented lower parameters than the control mayonnaise, this reduction was less than $20 \%$, comparing the control mayonnaise, evidencing with these results that, mucilage of chia, acts as an emulsifying agent, however the addition of $0.21 \mathrm{~g}$ of lyophilized chia mucilage is required to provide the $15 \%$ reduction of oil in mayonnaises, enhancing the texture parameters.

\section{Color}

The color parameters are the first criterion measured in the acceptance or rejection of the product by the consumer. Table 3 presents the color parameters of mayonnaises where the soybean oil was replaced by lyophilized chia mucilage at different percentages.

According to Table 3 , the luminosity value $\left(\mathrm{L}^{*}\right)$ was higher in the formulation control, while the formulations MOCM-A and MOCM-B did not differ statistically each other and were significantly higher than MOCM-C formulation, showing that the addition of chia mucilage 
Table 3. Color parameters of control mayonnaise and mayonnaises with replacement of oil by lyophilized chia mucilage

\begin{tabular}{|c|c|c|c|c|}
\hline Formulation & $\mathbf{L}^{*}$ & $\mathbf{a}^{*}$ & $\mathbf{b}^{*}$ & $\mathbf{h}\left(^{\mathbf{o}}\right)$ \\
\hline Control & $79.95 \pm 1.07^{\mathrm{a}}$ & $-1.01 \pm 0.50^{\mathrm{a}}$ & $37.72 \pm 1.46^{\mathrm{a}}$ & 88.46 \\
\hline MOCM-A & $71.20 \pm 1.60^{\mathrm{b}}$ & $-3.31 \pm 0.07^{\mathrm{c}}$ & $19.81 \pm 0.17^{\mathrm{b}, \mathrm{c}}$ & 80.51 \\
\hline MOCM-B & $70.05 \pm 0.45^{\mathrm{b}}$ & $-3.25 \pm 0.03^{\mathrm{b}, \mathrm{c}}$ & $20.80 \pm 0.15^{\mathrm{b}}$ & 81.11 \\
\hline MOCM-C & $66.13 \pm 1.13^{\mathrm{c}}$ & $-2.64 \pm 0.04^{\mathrm{b}}$ & $18.44 \pm 0.31^{\mathrm{c}}$ & 81.85 \\
\hline
\end{tabular}

MOCM-A, MOCM-B and MOCM-C: formulations with $0.5,1.0$ and 1,5\% addition of lyophilized mucilage in relation the $15 \%$ reduced of oil, respectively. L*: brightness; a* and $b^{*}$ : chroma; h: hue angle. Average of three values with standard deviation, same letter in the column indicates that there is no significant difference between the means by Tukey test $(\mathrm{p}<0.05)$

could influence mayonnaise color. The decrease in the luminosity $\left(\mathrm{L}^{*}\right)$ have been caused by the chia mucilage which has a darker coloration when rehydrated. Amim et al. [19] obtained an increase of the luminosity when they developed mayonnaises with low-fat containing xanthan gum, guar gum and a combination of xanthan and guar gums.

The parameter $\mathrm{a}^{\star}$, on the other hand, tended to green for the control mayonnaise as well as for mayonnaise with chia mucilage, due to the increase in the amount of chia mucilage added. The Hue angle is close to $90^{\circ}$, confirming the yellow coloration, as well as the parameter $\mathrm{b}^{*}$, which was positive (yellow) in all the formulations and was smaller than control mayonnaise.

\section{Conclusion}

Combining the characteristics of texture, $\mathrm{pH}$, water activity and color, it is concluded that the addition of $1 \%(0.21 \mathrm{~g})$ of lyophilized chia mucilage is the ideal quantity to simulate $15 \%$ ( $21 \mathrm{~g}$ ) of soybean oil in mayonnaise. Thus, it was verified that the emulsifying capacity of the mucilage of chia is 100 times greater than that of the soybean oil in mayonnaises, evidencing that the mucilage of chia can be applied in several foods as a substitution of fat, reducing the lipid content and maintaining the characteristics of the product.

\section{References}

1. Serin S, Sayar S (2017) The effect of the replacement of fat with carbohydrate-based fat replacers on the dough properties and quality of the baked pogaca: a traditional high-fat bakery product. Food Sci Technol 37: 25-32.

2. Zahn S, Pepke F, Rohm H (2010) Effect of inulin as a fat replacer on texture and sensory properties of muffins. Int J Food Sci Technol 45: 2531-2537.

3. Ma Z, Boye JI (2013) Advances in the Design and Production of Reduced-Fat and Reduced-Cholesterol Salad Dressing and Mayonnaise: A Review. Food Bioprocess Technol 6: 648-670.

4. Fernandes SS, Salas-Mellado ML2 (2017) Addition of chia seed mucilage for reduction of fat content in bread and cakes. Food Chem 227: 237-244. [Crossref]

5. Felisberto MHF, Wahanik AL, Gomes-Ruffi CR, Clerici MTPS, Chang YK, et al. (2015) Use of chia (Salvia hispanica L.) mucilage gel to reduce fat in pound cakes. Food Sci Technol 63: 1049-1055.
6. Capitani MI, Spotorno V, Nolasco SM, Tomás MC (2012) Physicochemical and functional characterization of by-products from chia (Salvia hispanica L.) seeds from Argentina. Food Sci Technol 45: 94-102.

7. Ali NM, Yeap SK, Ho WY, Beh BK, Tan SW, Tan SG (2012) The Promising Future of Chia, Salvia hispanica L. BioMed Research International 2012: 1-9.

8. Lin KY, Daniel JR (1994) Structure of chia seed polysaccharide exudates. Carbohydr Polym 23: 13-18.

9. Spada JC, Dick M, Pagno CH, Vieira AC, Bernstein A, et al. (2014) Caracterização física, química e sensorial de sobremesas à base de soja, elaboradas com mucilagem de chia. Cienc Rural 44: 374-379.

10. Campos BE, Ruivo TD, Scapim MRS, Madrona GS, Bergamasco RC (2016) Optimization of the mucilage extraction process from chia seeds and application in ice cream as a stabilizer and emulsifier. Food Sci Technol 65: 874-883.

11. Salgado-Cruz M de la P, Ramírez-Miranda M, Díaz-Ramírez M, Alamilla- Beltran L, Calderón-Domínguez G (2017) Microstructural characterisation and glycemic index evaluation of pita bread enriched with chia mucilage. Food Hydrocoll 69: 141-149.

12. Borneo R, Aguirre A, Leon AE (2010) Chia (Salvia hispanica L) gel can be used as egg or oil replacer in cake formulations. J Am Diet Assoc 110: 946-949. [Crossref]

13. Menga V, Amato M, Phillips TD, Angelino D, Morreale F, et al. (2017) Gluten-free pasta incorporating chia (Salvia hispanica L.) as thickening agent: An approach to naturally improve the nutritional profile and the in vitro carbohydrate digestibility. Food Chem 221: 1954-1961.

14. Ma Z, Boye JI (2013) Advances in the Design and Production of Reduced-Fat and Reduced-Cholesterol Salad Dressing and Mayonnaise: A Review. Food Bioprocess Tech 6: 648-670.

15. Depree JA, Savage GP (2001) Physical and flavour stability of mayonnaise. Food Sci Technol 12: 157-163.

16. Raikos V, Mcdonagh A, Ranawana V, Duthie G (2016) Processed beetroot (Beta vulgaris $\mathrm{L}$.) as a natural antioxidant in mayonnaise: Effects on physical stability, texture and sensory attributes. Food Science and Human Wellness 5: 191-198.

17. [Kishk YFM, Elsheshetawy HE (2013) Effect of ginger powder on the mayonnaise oxidative stability, rheological measurements, and sensory characteristics. Annals of Agricultural Science 58: 213-220.

18. Mun S, Kim YL, Kang CG, Park KH, Shim JY, et al. (2009) Development of reducedfat mayonnaise using 4alphaGTase-modified rice starch and xanthan gum. Int J Biol Macromol 44: 400-407. [Crossref]

19. Amim MHH, Elbeltagy AE, Mustafa M, Khalil AH (2014) Development of low fat mayonnaise containing different types and levels of hydrocolloid gum. J Agroaliment Proc Technol 20: 54-63.

20. Li J, Wang Y, Jin W, Zhou B (2014) Application of micronized konjac gel for fat analogue in mayonnaise. Food Hydrocoll 35: 375-382.

21. Gaonkar G, Koka R, Chen K, Cambell B (2010) Emulsifying functionality of enzymemodified milk proteins in $\mathrm{O} / \mathrm{W}$ and mayonnaise-like emulsions. Afr J Food Sci 1: 1625 .

22. Capitani MI, Nolasco SM, Tomás MC (2016) Stability of oil-in-water (O/W) emulsions with chia (Salvia hispanica L.) mucilage. Food Hydrocoll 61: 537-546.

23. Olsson V, Håkansson A, Purhagen J, Wendin K (2018) The Effect of Emulsion Intensity on Selected Sensory and Instrumental Texture Properties of Full-Fat Mayonnaise. Foods 2018: 7-9.

Copyright: (C2018 Fernandesa SS. This is an open-access article distributed under the terms of the Creative Commons Attribution License, which permits unrestricted use, distribution, and reproduction in any medium, provided the original author and source are credited. 